\title{
Longitudinal MRI evidence for decreased survival among periventricular glioblastoma
}

\author{
Geoffrey S. Young • Eric A. Macklin • Kian Setayesh • \\ Joshua D. Lawson - Patrick Y. Wen - Andrew D. Norden • \\ Jan Drappatz $\cdot$ Santosh Kesari
}

Received: 9 September 2010/Accepted: 17 November 2010/Published online: 5 December 2010

(C) The Author(s) 2010. This article is published with open access at Springerlink.com

\begin{abstract}
While the prognosis of patients with glioblastoma (GBM) remains poor despite recent therapeutic advances, variable survival times suggest wide variation in tumor biology and an opportunity for stratified intervention. We used volumetric analysis and morphometrics to measure the spatial relationship between subventricular zone (SVZ) proximity and survival in a cohort of 39 newly diagnosed GBM patients. We collected T2-weighted and gadolinium-enhanced T1-weighted magnetic resonance images (MRI) at pre-operative, post-operative, pre-radiation therapy, and post-radiation therapy time points, measured tumor volumes and distances to the SVZ, and collected clinical data. Univariate and multivariate Cox regression showed that tumors involving the SVZ and tumor growth rate during radiation therapy were independent predictors of shorter progression-free and overall
\end{abstract}

Geoffrey S. Young, Eric A. Macklin, and Kian Setayesh are co-first authors for this article.

Electronic supplementary material The online version of this article (doi:10.1007/s11060-010-0477-1) contains supplementary material, which is available to authorized users.

\section{G. S. Young $(\bowtie) \cdot$ K. Setayesh}

Department of Radiology, Division of Neuroradiology,

Brigham and Women's Hospital, 75 Francis Street,

Boston, MA 02115, USA

e-mail: gsyoung@partners.org

\section{E. A. Macklin}

Biostatistics Center, Massachusetts General Hospital,

50 Staniford Street, Boston,

MA 02114, USA

P. Y. Wen · A. D. Norden · J. Drappatz

Center for Neuro-Oncology, Dana-Farber Cancer Institute,

44 Binney Street, Boston, MA 02115, USA survival. These results suggest that GBMs in close proximity to the ependymal surface of the ventricles convey a worse prognosis-an observation that may be useful for stratifying treatment.

Keywords SVZ - Glioblastoma - Stem cell · Outcome · MRI

\section{Introduction}

Glioblastoma (GBM), the most frequent malignant glioma in adults [1] conveys a grim outlook, with median overall survival of 12-14 months from diagnosis. Prognosis and response to standard therapy with resection, radiation, and chemotherapy (temozolomide) are highly variable, so it would be desirable to identify subgroups of patients with poorer prognosis who may benefit from alternative or more aggressive chemotherapy and radiation regimens at the time of diagnosis. Patient age and performance status are long established clinical prognostic factors in GBM patients [2], but of the many molecular and imaging markers investigated

P. Y. Wen · A. D. Norden · J. Drappatz

Department of Neurology, Brigham and Women's Hospital,

75 Francis Street, Boston, MA 02115, USA

G. S. Young - E. A. Macklin · P. Y. Wen .

A. D. Norden · J. Drappatz

Harvard Medical School, 25 Shattuck Street,

Boston, MA 02115, USA

J. D. Lawson · S. Kesari $(\bowtie)$

Department of Neurosciences, Moores UCSD Cancer Center,

University of California, 3855 Health Sciences Drive,

MC 0819, La Jolla, CA 92093-0819, USA

e-mail: skesari@ucsd.edu 
for sub-phenotyping, only a few such as $\mathrm{O}^{6}$-methylguanineDNA methyltransferase (MGMT) promoter methylation status are predictive of response to chemoradiation in a retrospective studies and none has been validated prospectively [3]. Biomarkers predictive of prognosis are greatly needed to help individualize therapy of GBM.

Recent papers have suggested that GBM adjacent to the subventricular zone (SVZ) are more likely to manifest the highly invasive and multifocal phenotype and may be associated with increased morbidity $[4,5]$. Based on this, we hypothesized that GBM adjacent to the SVZ may have worse outcomes. We explored this hypothesis by longitudinally evaluating the size and location of tumors and correlating these measures with overall and progressionfree survival in a retrospective sample of newly diagnosed GBM patients.

\section{Patients and methods}

Patient population

Our hospital institutional review board approved this study. Consecutive glioblastoma (GBM, grade IV glioma) patients confirmed by surgical pathology at our hospital who presented initially between March 2003 and August 2005 were retrospectively identified through an institutional database. We identified 493 patients with diagnosis of glioma of any type. Of these, 75 patients had pathological diagnosis of GBM. Of these, 36 patients were excluded due to inadequate imaging or clinical follow-up; leaving 39 local patients whose pre-operative, post-operative, pre-radiation therapy and post-radiation therapy MRI scans could be obtained for analysis. All patients had undergone resection followed by involved-field radiation therapy (RT) with concurrent temozolomide and adjuvant temozolomide. Patients were monitored for progression and survival through February 2008. No patient was treated with bevacizumab.

Imaging data collection and post-processing

MRI was performed on 1.5 or 3.0 Tesla scanners (GE Medical Systems, Milwaukee, WI) using standard institutional brain tumor imaging protocols. At a uniform delay following intravenous administration of $20 \mathrm{cc}$ of gadopentate dimeglumine, T1-weighted spin-echo axial images (GdT1WI) were acquired with $\mathrm{TR}=417-550 \mathrm{~ms}$, $\mathrm{TE}=20 \mathrm{~ms}$, slice thickness $=5 \mathrm{~mm}$, and matrix $=256^{2}$ pixels. Fast spin echo (FSE) T2-weighted images (T2WI) with slice thickness $=5 \mathrm{~mm}$, and matrix $=256^{2}-512^{2}$ pixels were acquired using either pre-contrast fluid attenuated inversion recovery (FLAIR) technique with
$\mathrm{TR}=125-133 \mathrm{~ms}, \mathrm{TE}=8,002 \mathrm{~ms}$, or conventional FSE technique with $\mathrm{TR}=99-102 \mathrm{~ms}$, TE $=4,250-5,300 \mathrm{~ms}$. Tumor volumes were calculated using manual segmentation software (ITK-SNAP, Chapel Hill, NC).

Measurements

Tumor volumes and interval growth rates

$G d V$ and $T 2 V$ (volumes): Volume of abnormal enhancement $(\mathrm{GdV})$ was measured on GdT1WI. Volume of abnormal $\mathrm{T} 2$ prolongation $(\mathrm{T} 2 \mathrm{~V})$ was measured from FLAIR images in 66 scans and conventional FSET2WI in the 41 scans lacking diagnostic-quality FLAIR images.

$\triangle G d V$ and $\triangle T 2 V$ (volumetric growth rates): Enhancing tumor growth rate $(\Delta \mathrm{GdV})$ and non-enhancing tumor growth rate $(\Delta \mathrm{T} 2 \mathrm{~V})$ were calculated between pre-operative and post-operative and between pre-radiation and post-radiation scans by subtracting the earlier volume from the later volume and dividing the resulting change in volume by the number of days between the two scans.

\section{Tumor distances from the ventricles}

GdED and T2ED (edge distances): Distance from the edge of the abnormality to the ependyma of the ventricles was measured on GdT1WI (GdED) and T2WI images (T2ED) (Fig. 1). If the abnormality extended to the ependymal surface of the ventricle, this was noted as GdED $=0$ or $\mathrm{T} 2 \mathrm{ED}=0$.

$G d C D$ and $T 2 C D$ (center distances): Distance from the geometric center of abnormality to the ventricle was measured on GdT1WI (GdCD) or T2WI (T2CD). The geometric center was defined as the intersection of the largest in-plane anteroposterior and orthogonal diameters of contrast enhancement or T2 prolongation on the axial sections demonstrating the largest area of abnormality on GdT1WI or T2WI respectively (Fig. 1).

Distances (GdED, GdCD, T2ED, and T2CD) were measured in millimeters using FDA-approved commercial clinical DICOM viewing software (GE Centricity, Milwaukee WI).

\section{Tumor location}

Using the geometric center of enhancement on the preoperative GdT1WI, tumors were categorized as anterior (A) or posterior $(\mathrm{P})$ relative to the central sulcus and left (L) or right (R) relative to the interhemispheric fissure. Tumors centered in the temporal lobe were classified in the P subgroup. 
Fig. 1 Measurement of T2 volume ( $T 2 \mathrm{~V}$; left upper frame-thick red border), enhancing volume $(G d V$; right upper frame-thick red border), T2 edge distance (T2ED; left lower frame-radial yellow line from tumor edge to ventricle), enhancing edge distance (GdED; right lower frameradial yellow line from tumor edge to ventricle), T2 center distance $(T 2 C D$; left upper and lower frames-green line from centroid to ventricle), and enhancing center distance $(G d C D$; right upper and lower frames-left upper and lower frames-green line from centroid to ventricle) for patients with $\mathrm{T} 2 \mathrm{ED}=0$ and GdED $=0$ (upper) and T2ED $>0$ and GdED $>0$. (Color figure online)
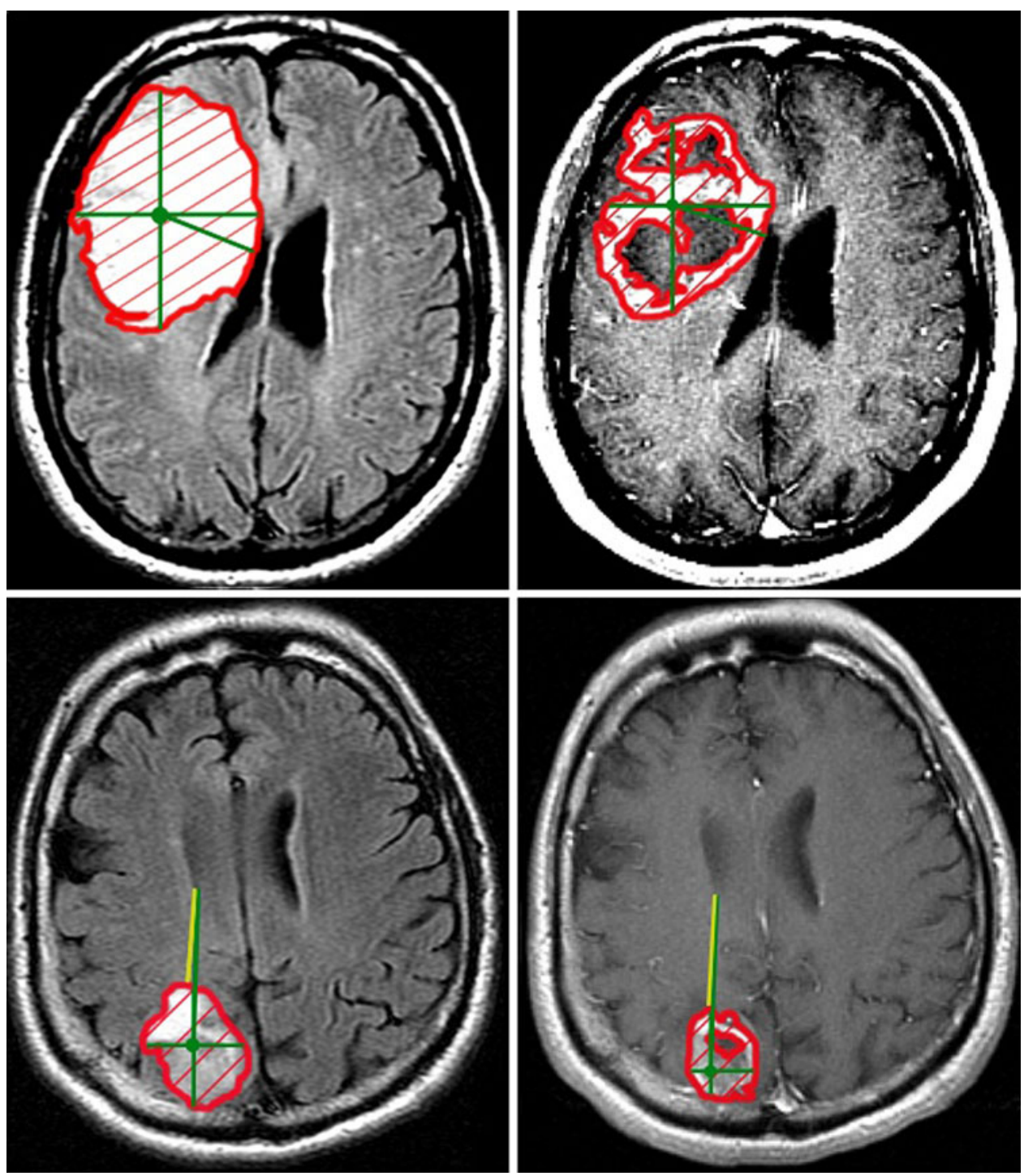

Surgical extent

When operative notes documented biopsy without attempt at definitive resection, the surgery was classified as a biopsy. If resection was attempted, the extent of resection was classified by analysis of the amount of abnormal enhancing tissue remaining on the post-operative GdT1WI using recently published methods [6]: detection of $>10 \mathrm{~cm}^{3}$ led to classification as subtotal resection (STR) and $\leq 1.0 \mathrm{~cm}^{3}$ as gross total resection (GTR).

Assessment of multifocality

Brain MRI images acquired at initial presentation and last follow-up and all spine MRI scans were assessed by two expert reviewers (GY,SK) for evidence of multifocal glioma at presentation and/or cerebrospinal fluid (CSF) spread during the course of therapy. Tumors having loci of abnormal enhancement separated by more than $2 \mathrm{~cm}$ on the initial pre-operative study were classified as multifocal [7]. Some reports have attempted to distinguish between multifocal glioma, presumed to represent early spread of a single lesion, and multicentric glioma, presumed to represent lesions that arose at multiple locations at one time [17]. Because the distinction is difficult to make in practice and recent analyses have demonstrated no difference in time to progression or survival between these groups [16], we did not attempt to distinguish between these putative subtypes. CSF spread was defined as occurrence of at least one new non-contiguous locus of abnormal enhancing tumor arising along the ependyma of the ventricles, pia covering the brain or spinal cord, or elsewhere in the subarachnoid space after the initial scan [4].

\section{Statistical analysis}

Patients in the study cohort were compared to those excluded from analysis with respect to age, Karnofsky performance 
status (KPS), and sex by $t$-test, Wilcoxon rank sum test, and Fisher's exact test, respectively. Overall survival (OS) and progression-free survival (PFS) were calculated in months from the pre-operative MRI. Progression was defined using Macdonald criteria [8]. The OS and PFS curves and median survival times were calculated as Kaplan-Meier productlimit estimates. Associations between OS and PFS and the following independent variables were analyzed by logrank test and univariate and multivariate Cox regression: age, sex, KPS, tumor location, duration of radiation therapy, extent of surgical resection, multifocality, CSF spread, GdV, T2V, $\Delta \mathrm{GdV}, \Delta \mathrm{T} 2 \mathrm{~V}, \mathrm{GdCD}, \mathrm{T} 2 \mathrm{CD}, \mathrm{GdED}$, and T2ED. The univariate analyses were considered exploratory. Reported $P$ values were not corrected for multiple comparisons. Multivariate models were identified by best subset selection with model complexity chosen by Bayesian information criterion. Terms for age (both constant and time-dependent) and other previously identified predictors of outcome (extent of resection, multifocality, CSF spread, and pre-operative tumor volume) were included in all models. Optimal models constructed from baseline predictors were selected prior to incorporating measures assessed after baseline. Due to our model selection procedure, the nominal $P$-values may be lower than would be estimated from an independent data set.

\section{Results}

Patient population, tumor characteristics, and survival

The study cohort included 29 male and 10 female patients. Mean age was 58 years (range: 27-81), median KPS 70, and median follow-up time 18.2 months (range, 3.1-41.7). Nineteen patients (49\%) had posterior tumors, including 7 temporal lobe tumors (37\% of the P subgroup, $18 \%$ overall). Thirty-three patients (85\%) underwent biopsy and 6 patients (15\%) STR. Four patients (10\%) had multifocal tumor at presentation. CSF spread was detected during follow-up in 4 patients $(10 \%)$. On the pre-operative scan, signal abnormality extended to the ependyma of the ventricles on T2WI $(\mathrm{T} 2 \mathrm{ED}=0)$ in $30 / 39$ patients $(77 \%)$ and on GdT1WI $($ GdED $=0)$ in $16 / 39$ patients $(41 \%)$ (Table 1). Four patients were alive at the end of follow-up. Median post-operative OS was 15.6 months (95\% CI: 11.1-24.1, Fig. 2) and median post-operative PFS 6.7 months (95\% CI: 5.2-7.2, Fig. 3). The 36 patients who were excluded had similar baseline features: age $($ median $=54$ years, $P=0.12$ ), KPS $($ median $=70$, $P=0.67)$, and sex $($ male $=64 \%, P=0.45)$.

Overall survival analysis

Univariate Cox regression identified older patient age and lower KPS as significant predictors of poor overall
Table 1 Patient and tumor characteristics $(n=39)$

\begin{tabular}{|c|c|}
\hline Variable & $\begin{array}{l}\text { Summary } \\
(\text { mean } \pm \mathrm{SD} \text { [range] or } n[\%])\end{array}$ \\
\hline Age (years) & $58.4 \pm 11.9(27.7,81.8)$ \\
\hline KPS & $74.4 \pm 10.5(60,100)$ \\
\hline Gender (male) & $29(74 \%)$ \\
\hline Brain region (posterior) & $19(49 \%)$ \\
\hline Hemisphere (left) & $19(49 \%)$ \\
\hline Resection extent (biopsy) & $33(85 \%)$ \\
\hline Multifocal at diagnosis & $4(10 \%)$ \\
\hline CSF dissemination & $4(10 \%)$ \\
\hline RT duration (months) & $2.74 \pm 0.54(1.97,4.93)$ \\
\hline Pre-Op T2V $\left(\mathrm{mm}^{3}\right)$ & $113 \pm 62(5.36,241)$ \\
\hline Pre-Op GdV $\left(\mathrm{mm}^{3}\right)$ & $35.7 \pm 25.8(0.106)^{2}$ \\
\hline Pre-RT T2V $\left(\mathrm{mm}^{3}\right)$ & $108 \pm 62(12.6,260)$ \\
\hline Pre-RT GdV $\left(\mathrm{mm}^{3}\right)$ & $41.2 \pm 27.8(4.10,117)$ \\
\hline Post-RT T2V $\left(\mathrm{mm}^{3}\right)$ & $126 \pm 102(0.711,437)$ \\
\hline Post-RT GdV $\left(\mathrm{mm}^{3}\right)$ & $32.0 \pm 24.8(0.550,101)^{\mathrm{a}}$ \\
\hline Pre-Op T2ED (mm) & $2.29 \pm 5.71(0.27 .1)$ \\
\hline Pre-Op GdED (mm) & $7.27 \pm 10.5(0.41 .4)$ \\
\hline Pre-RT T2ED (mm) & $1.80 \pm 4.71(0.24 .8)$ \\
\hline Pre-RT GdED (mm) & $6.15 \pm 8.52(0.26 .5)$ \\
\hline Post-RT T2ED (mm) & $0.701 \pm 2.32(0.11 .4)$ \\
\hline Post-RT GdED (mm) & $5.41 \pm 7.72(0.26 .6)$ \\
\hline Pre-Op T2ED $=0$ (yes vs. no) & $30(77 \%)$ \\
\hline Pre-Op GdED $=0$ (yes vs. no) & $16(41 \%)$ \\
\hline Pre-RT T2ED $=0$ (yes vs. no) & $30(77 \%)$ \\
\hline Pre-RT GdED $=0$ (yes vs. no) & $15(39 \%)$ \\
\hline Post-RT T2ED $=0$ (yes vs. no) & $35(90 \%)$ \\
\hline Post-RT GdED = 0 (yes vs. no) & $16(41 \%)$ \\
\hline Pre-Op T2CD (mm) & $20.7 \pm 8.0(9.58,37.8)$ \\
\hline Pre-Op GdCD (mm) & $28.2 \pm 11.0(8.76,50.5)$ \\
\hline Pre-RT T2CD (mm) & $22.5 \pm 10.5(3.39,48.8)$ \\
\hline Pre-RT GdCD (mm) & $26.8 \pm 11.3(9.33,55.5)$ \\
\hline Post-RT T2CD (mm) & $17.8 \pm 7.7(3.92,31.8)$ \\
\hline Post-RT GdCD (mm) & $24.3 \pm 10.5(8.90,50.1)$ \\
\hline Pre-Op $\rightarrow$ Pre-RT $\Delta \mathrm{T} 2 \mathrm{~V}\left(\mathrm{~mm}^{3} /\right.$ day $)$ & $-1.45 \pm 4.92(-16.0,12.6)$ \\
\hline Pre-Op $\rightarrow$ Pre-RT $\Delta \mathrm{GdV}\left(\mathrm{mm}^{3} /\right.$ day $)$ & $-0.215 \pm 5.21(-16.8,17.5)$ \\
\hline Pre $\rightarrow$ Post-RT $\Delta \mathrm{T} 2 \mathrm{~V}\left(\mathrm{~mm}^{3} /\right.$ day $)$ & $0.216 \pm 1.01(-0.940,3.07)$ \\
\hline Pre $\rightarrow$ Post-RT $\Delta \mathrm{GdV}\left(\mathrm{mm}^{3} /\right.$ day $)$ & $-0.100 \pm 0.271(-0.843,0.613)$ \\
\hline
\end{tabular}

${ }^{a}$ GdV data at the early and post-RT time points are based on 38 of 39 patients because T1WI scans were incompatible with the segmentation software

Pre-Op before biopsy or resection, Pre-RT after surgery and before radiation therapy, Post- $R T$ after radiation therapy, $T 2 \mathrm{~V}$ volume of abnormal T2 prolongation on FLAIR/FSE T2WI, $G d V$ volume of the tumor on GdT1WI, T2ED distance from the edge of the abnormality to the ventricles on FLAIR/FSE T2WI images, GdED distance from the edge of the abnormality to the ventricles on GdT1WI, $T 2 C D$ distance from the center of the tumor to the ventricles on FLAIR/FSE T2WI, $G d C D$ distance from the center of the tumor to the ventricles on GdT1WI, $\triangle T 2 V$ the difference in volume divided by the number of days between FLAIR/FSE T2WI scans, $\Delta G d V$ the difference in volume divided by the number of days between GdT1WI scans, $T 2 E D=0$ signal abnormality extended to the ventricle on FLAIR/FSE T2WI, GdED = 0 signal abnormality extended to the ventricle on GdT1WI 

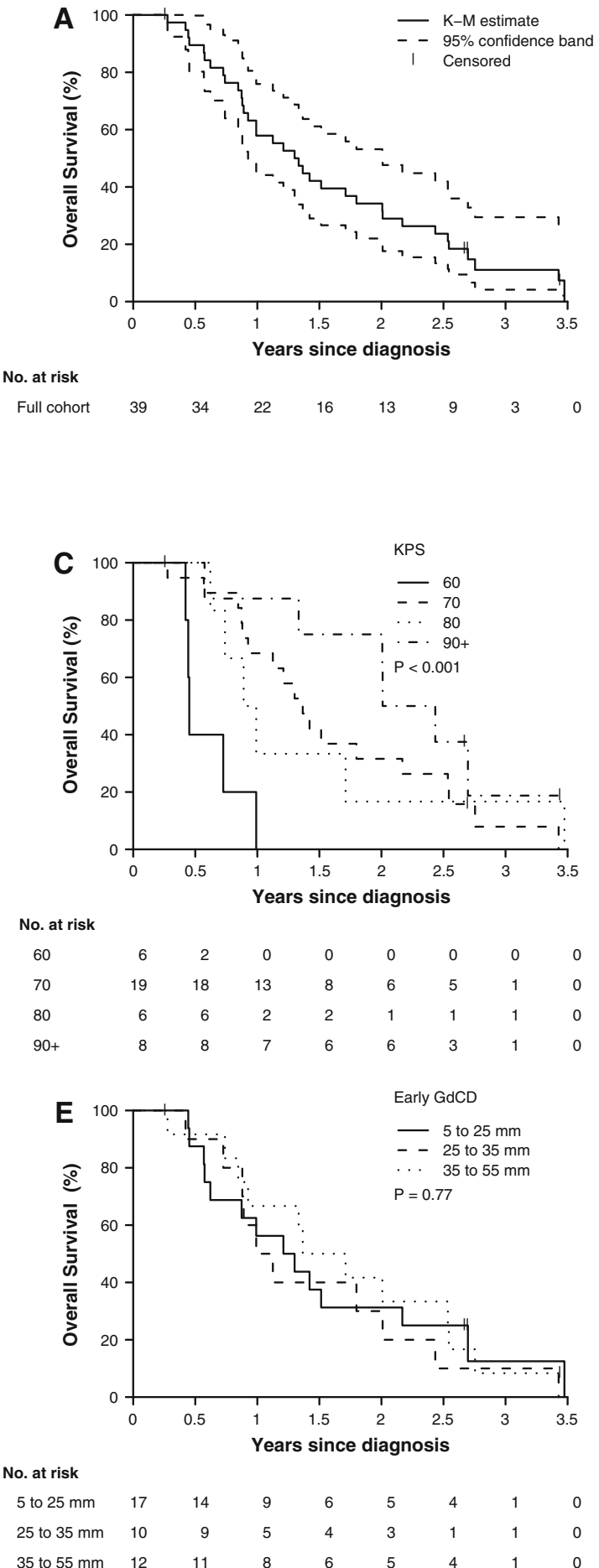

Fig. 2 Kaplan-Meyer plots depicting overall survival of the full cohort (a) and stratified by age (b), KPS (c), pre-operative SVZ involvement $(G d E D=0)(\mathbf{d})$, pre-operative geometric center

survival, with a $44 \%$ increase in mortality hazard for each 10 -year increment in age and a $40 \%$ increase in mortality hazard for each 10-unit decrease in KPS. Controlling for age and KPS, increased mortality hazard was associated with larger post-RT $\mathrm{T} 2 \mathrm{~V}, \Delta \mathrm{T} 2 \mathrm{~V} \geq 0$ over the course of $\mathrm{RT}$,

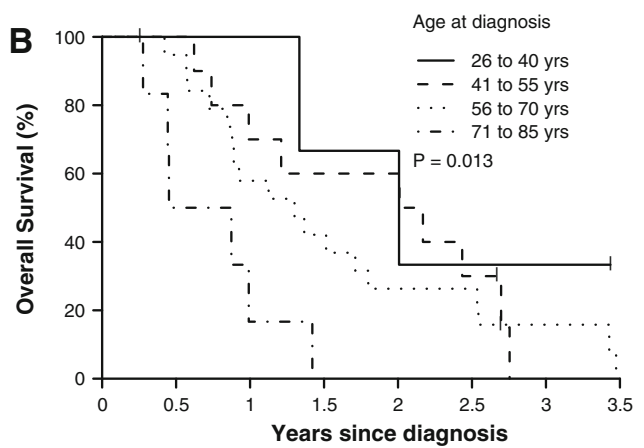

No. at risk

$\begin{array}{lrrrrrrrr}26 \text { to } 40 \text { yrs } & 3 & 3 & 3 & 2 & 2 & 1 & 1 & 0 \\ 41 \text { to } 55 \text { yrs } & 10 & 10 & 7 & 6 & 6 & 3 & 0 & 0 \\ 56 \text { to } 70 \mathrm{yrs} & 20 & 18 & 11 & 8 & 5 & 5 & 2 & 0 \\ 71 \text { to } 85 \mathrm{yrs} & 6 & 3 & 1 & 0 & 0 & 0 & 0 & 0\end{array}$

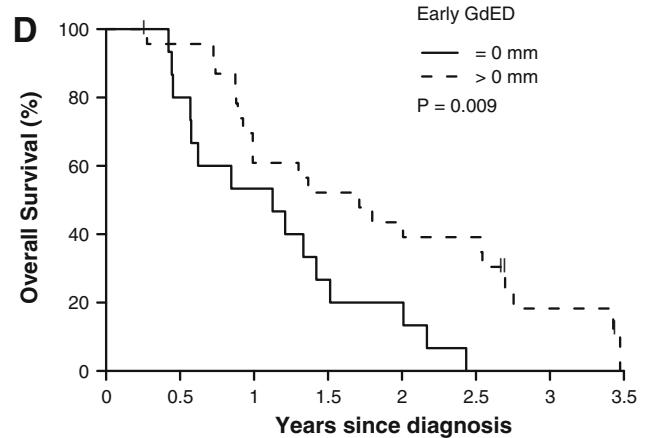

No. at risk

$\begin{array}{lllrrrrrr}0 \mathrm{~mm} & 16 & 12 & 8 & 4 & 3 & 0 & 0 & 0\end{array}$

$\begin{array}{llllllll}>0 & \mathrm{~mm} & 23 & 22 & 14 & 12 & 10 & 9\end{array}$

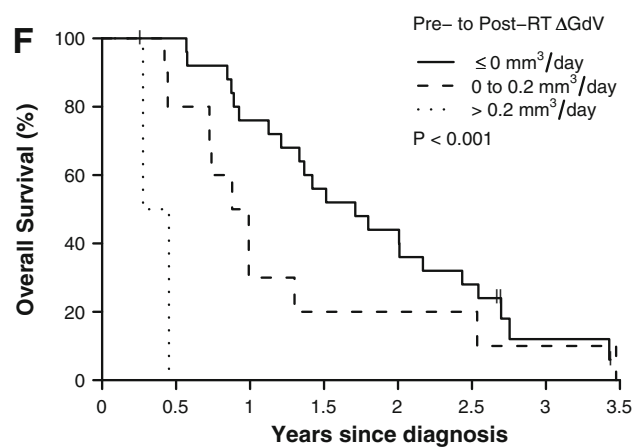

No. at risk

$\begin{array}{lrrrrrrrr}\leq 0 \mathrm{~mm}^{3} / \text { day } & 26 & 25 & 19 & 14 & 11 & 7 & 2 & 0 \\ 0 \text { to } 0.2 \mathrm{~mm}^{3} \text { /day } & 10 & 8 & 3 & 2 & 2 & 2 & 1 & 0 \\ >0.2 \mathrm{~mm}^{3} / \text { day } & 2 & 0 & 0 & 0 & 0 & 0 & 0 & 0\end{array}$

distance $(G d C D)(\mathbf{e})$, and change in enhancing tumor growth rate $(\Delta G d V)$ during radiation therapy (f). $P$-values from logrank tests

and tumor abutting the SVZ $(\mathrm{GdED}=0)$ (Supplementary Table 1).

Multivariate analysis of OS identified the known poor prognostic indicators of older age, lower KPS, and smaller extent of surgical resection as independent predictors of 

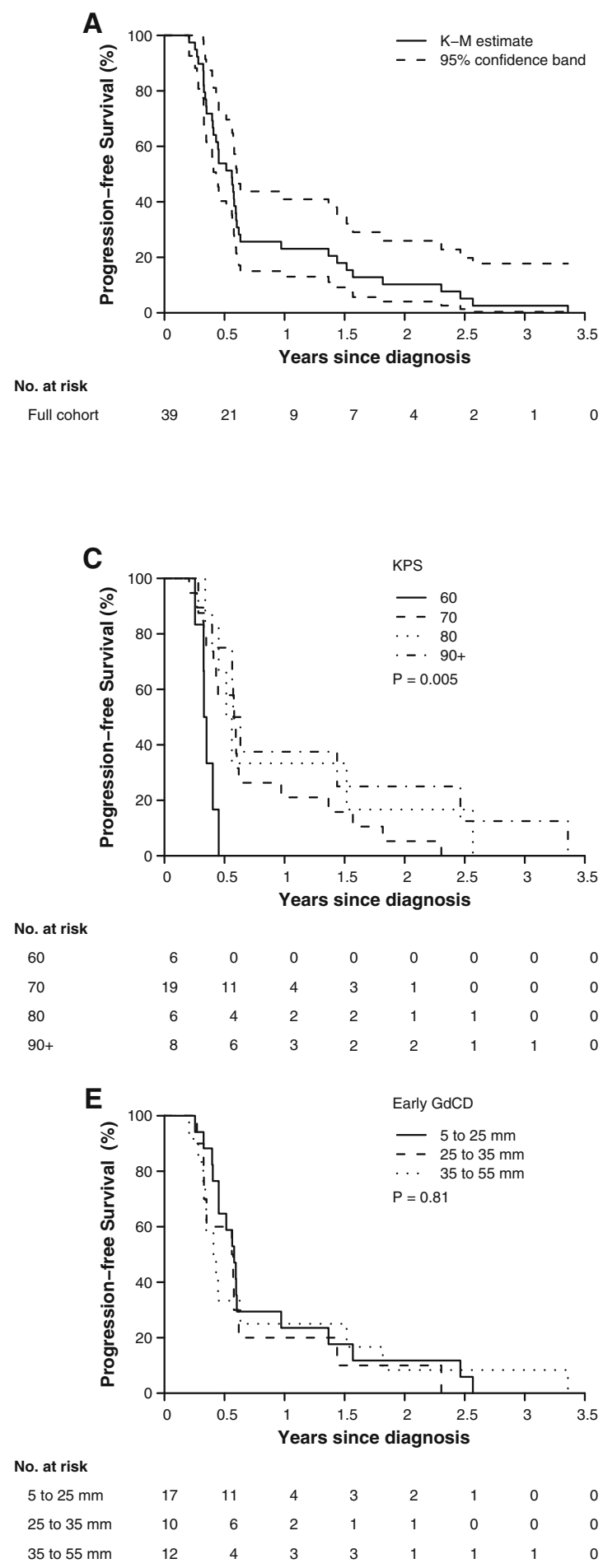

Fig. 3 Kaplan-Meyer plots depicting progression-free survival of the full cohort (a) and stratified by age (b), KPS (c), pre-operative SVZ involvement $(G d E D=0) \quad(\mathbf{d})$, pre-operative geometric center

increased mortality risk. Older patients were at higher risk of mortality initially, but analysis of time-dependent effect of age suggested that younger patients experienced accelerated risk of mortality relative to older patients during
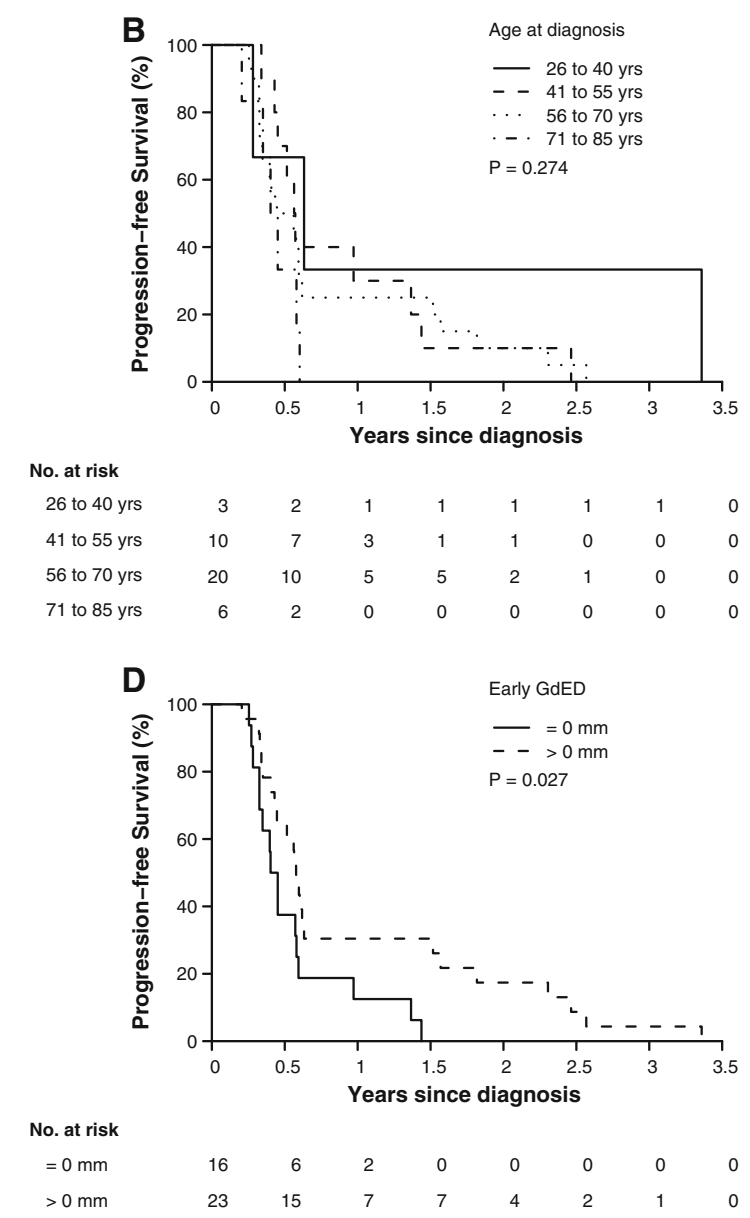

$\begin{array}{lrrrrrrrr}=0 \mathrm{~mm} & 16 & 6 & 2 & 0 & 0 & 0 & 0 & 0 \\ >0 \mathrm{~mm} & 23 & 15 & 7 & 7 & 4 & 2 & 1 & 0\end{array}$

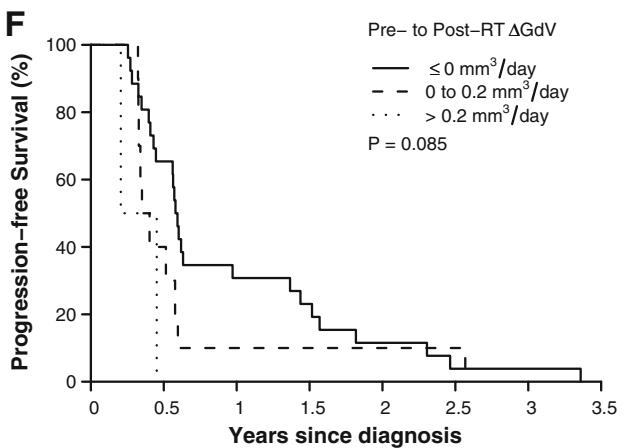

No. at risk

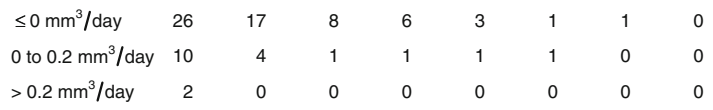

distance $(G d C D)(\mathbf{e})$, and enhancing tumor growth rate $(\Delta G d V)$ during radiation therapy (f). $P$-values from logrank tests

follow-up. Controlling for age and KPS, larger pre-operative $\mathrm{GdCD}$, larger positive $\triangle \mathrm{T} 2 \mathrm{~V}$ over the course of $\mathrm{RT}$, and pre-operative GdED $=0$ were independent predictors of mortality risk (Table 2). Pre-operative SVZ involvement 
Table 2 Multivariate Cox models of overall and progression-free survival

\begin{tabular}{|c|c|c|c|c|}
\hline \multirow[t]{2}{*}{ Variable } & \multicolumn{2}{|l|}{ Overall survival } & \multicolumn{2}{|l|}{ Progression-free survival } \\
\hline & Hazard ratio $(95 \% \mathrm{CI})$ & $P$-value & Hazard ratio $(95 \% \mathrm{CI})$ & $P$-value \\
\hline Age (per 10-year increment) & $3.15(1.33,7.46)$ & 0.009 & $1.30(0.67,2.55)$ & 0.44 \\
\hline Age (time-dependent, per month $\times 10$ years) & $0.42(0.22,0.82)$ & 0.010 & $0.51(0.23,1.13)$ & 0.098 \\
\hline KPS (per 10-unit increase) & $0.50(0.28,0.91)$ & 0.022 & $0.56(0.30,1.04)$ & 0.067 \\
\hline Resection extent (biopsy vs. subtotal) & $5.89(1.39,25.1)$ & 0.016 & $2.55(0.64,10.2)$ & 0.19 \\
\hline CSF dissemination (yes vs. no) & $0.96(0.23,4.02)$ & 0.95 & $1.27(0.37,4.33)$ & 0.70 \\
\hline Multifocal at diagnosis (yes vs. no) & $0.92(0.29,2.92)$ & 0.89 & $0.79(0.25,2.47)$ & 0.69 \\
\hline Pre-Op GdV (per $25 \mathrm{~mm}^{3}$ ) & $1.18(0.78,1.80)$ & 0.44 & $0.74(0.41,1.31)$ & 0.30 \\
\hline Pre-Op GdED = 0 (yes vs. no) & $3.70(1.41,9.73)$ & 0.008 & $4.24(1.47,12.2)$ & 0.008 \\
\hline Pre-Op GdCD (per $5 \mathrm{~mm}$ ) & $1.21(1.01,1.45)$ & 0.040 & $1.47(1.21,1.78)$ & $<0.0001$ \\
\hline Pre $\rightarrow$ Post-RT $\Delta \mathrm{T} 2 \mathrm{~V}$ (per $0.5 \mathrm{~mm}^{3} /$ day) & $1.38(1.11,1.73)$ & 0.004 & $1.39(1.11,1.75)$ & 0.004 \\
\hline
\end{tabular}

Note abbreviations in Table 1

$(\mathrm{GdED}=0)$ conveyed a nearly fourfold higher risk of mortality (hazard ratio: 3.70, 95\% CI: 1.41-9.73, $P=0.008)$ after adjustment for all other predictors of mortality risk. While the model controlled for pre-operative GdV and T2V, multifocal presentation, and CSF dissemination, these factors were not significant independent predictors of mortality risk. Inference was unchanged and point estimates changed by less than $2 \%$ when CSF dissemination and multifocality were dropped from the model.

Progression-free survival analysis

In the univariate analysis, age was not a significant predictor of PFS, but as with OS, a 10-unit decrease in KPS was associated with a $40 \%$ increase in hazard of progression or death. Larger pre-operative T2CD, larger pre-RT $\mathrm{T} 2 \mathrm{CD}$ and GdCD, larger post-RT T2V and GdV, $\Delta \mathrm{T} 2 \mathrm{~V} \geq 0$ over the course of RT, and pre-operative GdED $=0$ were associated with greater hazard of progression or death (Supplementary Table 1). After controlling for age and KPS, only larger pre-operative and pre-RT T2CD, and $\Delta \mathrm{T} 2 \mathrm{~V} \geq 0$ over the course of $\mathrm{RT}$, remained significant independent predictors of increased hazard of progression or death (Supplementary Table 1).

Age, KPS, and extent of surgical resection were not significant predictors of PFS in multivariate analysis. Larger pre-operative GdCD, $\Delta \mathrm{T} 2 \mathrm{~V} \geq 0$ over the course of $\mathrm{RT}$, and pre-operative GdED $=0$ were identified as independent predictors of risk of progression or mortality (Table 2). GdV prior to surgery, CSF dissemination, and multifocality were not found to be significant predictors of PFS. Pre-operative GdED $=0$ conveyed more than a fourfold higher risk of tumor progression or mortality (hazard ratio: $4.24,95 \% \mathrm{CI}: 1.47-12.2, P=0.008$ ) after adjustment for other predictors of progression and mortality risk. Inference was unchanged and point estimates changed by less than $5 \%$ when CSF dissemination and multifocality were dropped from the model.

\section{Discussion}

We have hypothesized that GBM in close proximity to the SVZ might be more malignant and invasive than GBM elsewhere. Our retrospective analysis is clearly not able to establish the origin of these GBM, but does argue for tumor proximity to the SVZ as an independent predictor for survival by demonstrating a worse prognosis in patients whose tumors extend to the ependymal surface of the ventricles on GdT1WI $(\mathrm{GdED}=0)$. The single previous report suggesting shorter survival in patients with GBM adjacent to the lateral ventricles was significantly limited by the heterogeneous extent of resection (35\% GTR) and application of optimal chemotherapy (27\% temozolomide) [9]. In contrast, the absence of GTR, and use of optimal chemotherapy in all patients in our cohort and the explicit statistical analysis for the confounding effect of STR versus biopsy described above make it very unlikely that the decreased survival of patients with periventricular tumors in this study was an artifact of incomplete surgical resection or suboptimal chemotherapy in patients with deeper tumors.

After controlling for the known prognostic variables of age, KPS, and extent of surgical resection, univariate analysis of OS, multivariate analysis of OS, and multivariate analysis of PFS all demonstrated increased risk of progression and mortality in our cohort for tumors having GdED $=0$ at presentation. Univariate analysis of the same association with PFS was not statistically significant at $P=0.065$. This reason that GBM adjacent to the ventricles on the initial GdT1WI conveys a poorer prognosis is not known. Others and we have hypothesized that more- 
aggressive GBM may arise in the SVZ, potentially from less differentiated progenitor or stem cells. While more patients had tumors extending to the ependymal surface of the ventricles on the T2WI $(\mathrm{T} 2 \mathrm{ED}=0)$ than on the GdT1WI (GdED = 0), OS and PFS were only associated with $\mathrm{GdED}=0$. This seems likely to reflect the fact that abnormality on T2WI represents a variable admixture of vasogenic edema and non-enhancing tumor whereas abnormality on GdT1WI may correlate more closely with the site of origin of the glioma.

Our analysis of confounding factors associated with deep tumors considered other possible effects, including greater difficulty of resection, greater involvement of deep white matter, and more common CSF spread. While no patient in our cohort underwent GTR, biopsy was associated with a two to six fold increase in risk of progression or mortality relative to STR. Nevertheless, after controlling for this variable, the presence of tumor adjacent to the ventricle on pre-operative GdT1WI images was independently predictive of worse OS and PFS. Patients with inoperable tumors of the brainstem, corpus callosum, left parietal lobe, and deep nuclei have been reported to have a poorer overall survival $[2,6,10]$, but no such tumors were observed in our cohort. Recent literature suggests that CSF spread has no significant effect on survival when compared to local tumor recurrence, but that multifocal enhancing tumor at presentation decreases survival significantly [4, 5]. The percentage of patients in our cohort with CSF spread and multifocality was similar to those reports. Although small sample size limits our power to detect these associations, neither factor predicted significantly shorter OS or PFS in our sample. Thus it seems unlikely that the association of tumor location in the SVZ with worse prognosis is an artifact of limited resection, involvement of deep white matter tracts, multifocality, or CSF spread. An additional factor that might confound the study is tumor genetics, which was beyond the scope of the current study.

In addition to the strong association with extension to the SVZ, larger pre-op GdCD and pre-RT T2CD were associated with increased risk of progression and mortality. Since the ventricle itself blocks medial spread, enlargement and white matter spread of tumors arising in the SVZ, will both necessarily displace the geometric center of tumor farther from the ventricle. Thus, T2CD and GdCD seem likely to represent surrogate markers for tumor size and/or extent of white matter spread. Although direct measures of larger tumor volume were associated with greater risk of mortality, the association disappeared after controlling for age, tumor growth rate, and GdED $=0$. Because an effect of tumor size on prognosis is suggested by recent $[11,12]$ but not older reports [10, 13], and our sample size is too small to allow a definitive conclusion, additional study is indicated to determine whether tumor size affects prognosis after controlling for tumor location in the SVZ.

In addition to acting as a surrogate for larger tumor size, greater pre-op and pre-RT GdCD and T2CD may reflect a more invasive phenotype. It seems likely that invasiveness of GBM at baseline and/or success of chemoradiation in controlling invasiveness may have a strong effect on survival. Although our study was not designed or powered to assess this hypothesis, we observed several associations consistent with this. Both univariate and multivariate analyses detected a significant association of tumor spread during $\mathrm{TMZ} / \mathrm{RT}(\Delta \mathrm{T} 2 \mathrm{~V} \geq 0)$ with decreased survival. Similarly, univariate analysis revealed that the extent of abnormality on T2WI after RT (T2V) was a significant predictor of decreased survival. Thus the possibility that change in extent of FLAIR/FSE T2WI abnormality during chemoradiation may be a useful surrogate for control of the infiltrative component of GBM. The possible influence of this marker on survival deserves further study. In this context, the fact that GdT1WI measurements of tumor growth during TMZ/RT and tumor size after RT were not significantly associated with survival, after adjusting for age and KPS, may reflect the greater efficacy of surgery and chemoradiation at controlling the enhancing focal component of GBM than the infiltrative component. Analysis in larger data sets including more sophisticated diffusion weighted imaging derived measures of white matter invasion, seems indicated to better resolve the independent effects of tumor volume, infiltrative tumor spread, and SVZ location at presentation.

Limitations of our study include the small sample size and our inability to control for molecular and genetic heterogeneity within GBM (including in particular MGMT methylation status), variability in post-progression salvage treatments, and longitudinal steroid dosing. Nonetheless, our findings illustrate that in addition to being a powerful research tool, quantitative imaging metrics characterizing tumor location and infiltrative spread of non-enhancing tumor deserve to be evaluated for stratifying patients for clinical trials and clinical care.

Our results suggest that patients with GBM extending to the SVZ, as defined by GdED $=0$ on the initial MRI, should be regarded as having an especially poor prognosis. After controlling for other known potential negative factors, tumors touching the SVZ continued to convey a worse prognosis. Neuropathologists have long postulated that certain types of GBM arise primarily from the SVZ [14, $15]$. Recent characterization of the oncogenic potential of SVZ stem cells has led to renewed interest in establishing a correlation between human glioma and the SVZ [16, 17]. Our series certainly does not establish this causative relationship, but is thought provoking in that we have found patients with GBM abutting the ventricles to have 
outcomes inferior to those whose tumors are some distance from the SVZ. This negative effect appears to be independent of other known factors influencing outcomes, though again genetic/epigenetic analysis, differences in vascularity, and other unknown factors were not evaluated in this series. As a first step, a prospective trial incorporating focal radiation of the SVZ could be considered within the sub-population of GBM patients whose tumor extends to the ependymal surface of the ventricles.

Open Access This article is distributed under the terms of the Creative Commons Attribution Noncommercial License which permits any noncommercial use, distribution, and reproduction in any medium, provided the original author(s) and source are credited.

\section{References}

1. Wen PY, Kesari S (2008) Malignant gliomas in adults. N Engl J Med 359(5):492-507

2. Mineo JF et al (2007) Prognosis factors of survival time in patients with glioblastoma multiforme: a multivariate analysis of 340 patients. Acta Neurochir (Wien) 149(3):245-252 Discussion: 252-253

3. Hegi ME et al (2005) MGMT gene silencing and benefit from temozolomide in glioblastoma. N Engl J Med 352(10):997-1003

4. Parsa AT et al (2005) Prognostic significance of intracranial dissemination of glioblastoma multiforme in adults. J Neurosurg 102(4):622-628

5. Showalter TN et al (2007) Multifocal glioblastoma multiforme: prognostic factors and patterns of progression. Int $\mathrm{J}$ Radiat Oncol Biol Phys 69(3):820-824

6. Stummer W et al (2008) Extent of resection and survival in glioblastoma multiforme: identification of and adjustment for bias. Neurosurgery 62(3):564-576 Discussion: 564-576
7. Lim DA et al (2007) Relationship of glioblastoma multiforme to neural stem cell regions predicts invasive and multifocal tumor phenotype. Neuro Oncol 9(4):424-429

8. Macdonald DR et al (1990) Response criteria for phase II studies of supratentorial malignant glioma. J Clin Oncol 8(7):1277-1280

9. Chaichana KL, McGirt MJ, Frazier J, Attenello F, GuerreroCazares H, Quinones-Hinojosa A (2008) Relationship of glioblastoma multiforme to the lateral ventricles predicts survival following tumor resection. J Neurooncol 89(2):219-224

10. Hammoud MA et al (1996) Prognostic significance of preoperative MRI scans in glioblastoma multiforme. J Neurooncol 27(1):65-73

11. Chow KL et al (2000) Prognostic factors in recurrent glioblastoma multiforme and anaplastic astrocytoma treated with selective intra-arterial chemotherapy. AJNR Am J Neuroradiol 21(3):471-478

12. Galanis E et al (2006) Validation of neuroradiologic response assessment in gliomas: measurement by RECIST, two-dimensional, computer-assisted tumor area, and computer-assisted tumor volume methods. Neuro Oncol 8(2):156-165

13. Wood JR, Green SB, Shapiro WR (1988) The prognostic importance of tumor size in malignant gliomas: a computed tomographic scan study by the Brain Tumor Cooperative Group. J Clin Oncol 6(2):338-343

14. Globus JH, Kuhlenbeck H (1942) Tumors of the striatothalamic and related regions: their probable source of origin and more common forms. Arch Pathol 34:674-734

15. Globus JH, Kuhlenbeck H (1944) The subependymal plate (matrix) and its relationship to brain tumors of the ependymal type. J Neuropath Exp Neurol 3:1-35

16. Sanai N, Alvarez-Buylla A, Berger MS (2005) Neural stem cells and the origin of gliomas. N Engl J Med 353(8):811-822

17. Bohman LE, Swanson KR, Moore JL, Rockne R, Mandigo C, Hankinson T, Assanah M, Canoll P, Bruce JN (2010) Magnetic resonance imaging characteristics of glioblastoma multiforme: implications for understanding glioma ontogeny. Neurosurgery 67(5):1319-1327 Discussion: 1327-1328 